\title{
Mean-field theory for the Mott-insulator-paired-superfluid phase transition in the two-species Bose-Hubbard model
}

\author{
M. Iskin \\ Department of Physics, Koç University, Rumelifeneri Yolu, TR-34450 Sariyer, Istanbul, Turkey
}

(Received 18 October 2010; published 12 November 2010)

\begin{abstract}
The standard mean-field theory for the Mott-insulator-superfluid phase transition is not sufficient to describe the Mott-insulator-paired-superfluid phase transition. Therefore, by restricting the two-species Bose-Hubbard Hamiltonian to the subspace of paired particles, and using perturbation theory, here we derive an analytic mean-field expression for the Mott-insulator-paired-superfluid transition boundary.
\end{abstract}

DOI: 10.1103/PhysRevA.82.055601

PACS number(s): 03.75.-b, 37.10.Jk, 67.85.-d

\section{INTRODUCTION}

Following the recent observation of Mott-insulatorsuperfluid phase transition with ultracold atomic Bose gases loaded into optical lattices [1-4], there has been intense theoretical activity in analyzing many Hubbard-type lattice models [5]. Among them the two-species Bose-Hubbard model, which can be studied with two-component Bose gases loaded into optical lattices, is one of the most popular. This is because, in addition to the Mott-insulator and single-speciessuperfluid phases, it has been predicted that this model has at least two additional phases: an incompressible super-counter flow and a compressible paired-superfluid phase [6-11].

Our main interest here is in the latter phase, where a direct transition from the Mott-insulator phase to the pairedsuperfluid phase (superfluidity of composite bosons, i.e., BoseBose pairs) has been predicted, when both species have integer fillings and the interspecies interaction is sufficiently large and attractive. In this paper, we derive an analytic mean-field expression for the Mott-insulator-paired-superfluid transition boundary in the two-species Bose-Hubbard model. The remaining paper is organized as follows. After introducing the model Hamiltonian in Sec. II, first we derive the mean-field theory in Sec. II A, and then present typical phase diagrams in Sec. II B. A brief summary of our conclusions is given in Sec. III.

\section{TWO-SPECIES BOSE-HUBBARD MODEL}

The two-species Bose-Hubbard Hamiltonian is given by

$$
\begin{aligned}
H= & -\sum_{i, j, \sigma} t_{i j, \sigma} b_{i, \sigma}^{\dagger} b_{j, \sigma}+\sum_{i, \sigma} \frac{U_{\sigma \sigma}}{2} \widehat{n}_{i, \sigma}\left(\widehat{n}_{i, \sigma}-1\right) \\
& +U_{\uparrow \downarrow} \sum_{i} \widehat{n}_{i, \uparrow} \widehat{n}_{i, \downarrow}-\sum_{i, \sigma} \mu_{\sigma} \widehat{n}_{i, \sigma},
\end{aligned}
$$

where the pseudospin $\sigma \equiv\{\uparrow, \downarrow\}$ labels the trapped hyperfine states of a given species of bosons or labels different types of bosons in a two-species mixture, $t_{i j, \sigma}$ is the tunneling (or hopping) matrix between sites $i$ and $j, b_{i, \sigma}^{\dagger}\left(b_{i, \sigma}\right)$ is the boson creation (annihilation) and $\widehat{n}_{i, \sigma}=b_{i, \sigma}^{\dagger} b_{i, \sigma}$ is the boson number operator at site $i, U_{\sigma \sigma^{\prime}}$ is the strength of the onsite boson-boson interaction between $\sigma$ and $\sigma^{\prime}$ components, and $\mu_{\sigma}$ is the chemical potential. In this paper, we consider a $d$-dimensional hypercubic lattice, for which we assume $t_{i j, \sigma}$ is a real symmetric matrix with elements $t_{i j, \sigma}=t_{\sigma} \geqslant 0$ for $i$ and $j$ nearest neighbors and 0 otherwise. We take the intraspecies interactions to be repulsive $\left(\left\{U_{\uparrow \uparrow}, U_{\downarrow \downarrow}\right\}>0\right)$ and the interspecies interaction to be attractive $\left(U_{\uparrow \downarrow}<0\right)$ such that $U_{\uparrow \uparrow} U_{\downarrow \downarrow}>U_{\uparrow \downarrow}^{2}$, to guarantee the stability of the mixture against collapse.

For sufficiently attractive $U_{\uparrow \downarrow}$, it is well established that [6-11] instead of a direct transition from the Mott insulator to a single-particle superfluid phase, the transition is from the Mott insulator to a paired-superfluid phase (superfluidity of composite bosons, i.e., Bose-Bose pairs). In fact, in the limit when $\left\{t_{\uparrow}, t_{\downarrow}\right\} \rightarrow 0$, it can be shown that the transition is from the Mott insulator to a paired-superfluid phase for all $U_{\uparrow \downarrow}<0$ [12].

\section{A. Mean-field theory}

In the single-species Bose-Hubbard model, the standard mean-field theory, where the boson creation and annihilation operators are approximated by their expectation values, e.g., $b_{i, \sigma}=\left\langle b_{i, \sigma}\right\rangle+\delta b_{i, \sigma}$, has proved to be very useful in understanding the qualitative features of the Mott-insulatorsingle-species-superfluid phase transition [5]. This is simply because the transition is driven by the first-order hopping effects. However, the Mott-insulator-paired-superfluid transition is driven by the second-order hopping effects, and therefore, the standard mean-field theory is not sufficient. This difficulty could be overcome by restricting the Hamiltonian to the subspace of paired particles, and including the secondorder hopping effects through second-order perturbation theory $[6-8,11]$.

For the Hamiltonian given in Eq. (1), we have recently calculated the two-particle and two-hole excitation energies (i.e., energy costs for adding and removing two particles, respectively) up to third order in the hoppings. Assuming $\left\{U_{\sigma \sigma},\left|U_{\uparrow \downarrow}\right|, 2 U_{\sigma \sigma}+U_{\uparrow \downarrow}\right\} \gg t_{\sigma}$, the two-particle excitation energy was found to be [12]

$$
\begin{aligned}
E_{\mathrm{p}}= & U_{\uparrow \downarrow}\left(n_{\uparrow}+n_{\downarrow}+1\right)+\sum_{\sigma}\left(U_{\sigma \sigma} n_{\sigma}-\mu_{\sigma}\right) \\
& +\sum_{\sigma}\left[\frac{\left(n_{\sigma}+1\right)^{2}}{U_{\uparrow \downarrow}}-\frac{n_{\sigma}\left(n_{\sigma}+2\right)}{2 U_{\sigma \sigma}+U_{\uparrow \downarrow}}+\frac{2 n_{\sigma}\left(n_{\sigma}+1\right)}{U_{\sigma \sigma}}\right] z t_{\sigma}^{2} \\
& +\frac{2\left(n_{\uparrow}+1\right)\left(n_{\downarrow}+1\right)}{U_{\uparrow \downarrow}} z t_{\uparrow} t_{\downarrow},
\end{aligned}
$$


where $z=2 d$ is the coordination number. Similarly, the twohole excitation energy was found to be [12]

$$
\begin{aligned}
E_{\mathrm{h}}= & -U_{\uparrow \downarrow}\left(n_{\uparrow}+n_{\downarrow}-1\right)-\sum_{\sigma}\left[U_{\sigma \sigma}\left(n_{\sigma}-1\right)-\mu_{\sigma}\right] \\
& +\sum_{\sigma}\left[\frac{n_{\sigma}^{2}}{U_{\uparrow \downarrow}}-\frac{\left(n_{\sigma}^{2}-1\right)}{2 U_{\sigma \sigma}+U_{\uparrow \downarrow}}+\frac{2 n_{\sigma}\left(n_{\sigma}+1\right)}{U_{\sigma \sigma}}\right] z t_{\sigma}^{2} \\
& +\frac{2 n_{\uparrow} n_{\downarrow}}{U_{\uparrow \downarrow}} z t_{\uparrow} t_{\downarrow},
\end{aligned}
$$

The accuracy of Eqs. (2) and (3) are checked via exact smallcluster (two-site) calculations. In addition, in the limit when $t_{\uparrow}=t_{\downarrow}=t, \quad U_{\uparrow \uparrow}=U_{\downarrow \downarrow}=U_{0}, \quad U_{\uparrow \downarrow}=U^{\prime}, \quad n_{\uparrow}=n_{\downarrow}=n_{0}$, $\mu_{\uparrow}=\mu_{\downarrow}=\mu$, and $z=2$ (or $d=1$ ), Eq. (3) is in complete agreement with Eq. (3) of Ref. [10], providing an independent check of the algebra. We note that, unlike the usual BoseHubbard model where $t_{\sigma}$ scales as $1 / d$ when $d \rightarrow \infty$, here $t_{\sigma}$ must scale as $1 / \sqrt{d}$ when $d \rightarrow \infty$.

Given the two-particle and two-hole excitation energies, the mean-field phase boundary for the Mott-insulator-pairedsuperfluid transition is determined by (see Ref. [11] for a similar calculation)

$$
1=\frac{c_{\mathrm{p}}}{E_{\mathrm{p}}+c_{\mathrm{p}}}+\frac{c_{\mathrm{h}}}{E_{\mathrm{h}}+c_{\mathrm{h}}},
$$

where $c_{\mathrm{p}}=-2\left(n_{\uparrow}+1\right)\left(n_{\downarrow}+1\right) z t_{\uparrow} t_{\downarrow} / U_{\uparrow \downarrow}$ and $c_{\mathrm{h}}=-2 n_{\uparrow} n_{\downarrow}$ $z t_{\uparrow} t_{\downarrow} / U_{\uparrow \downarrow}$. We note that, in the limit when $t_{\uparrow}=$ $t_{\downarrow}=J, U_{\uparrow \uparrow}=U_{\downarrow \downarrow}=U, U_{\uparrow \downarrow}=W \approx-U, n_{\uparrow}=n_{\downarrow}=m$, and $\mu_{\uparrow}=\mu_{\downarrow}=\mu$, Eq. (4) reduces to Eq. (5) of Ref. [11] (after setting $U_{N N}=0$ there). However, the terms that are proportional to $t_{\uparrow} t_{\downarrow}$ are not included in their definitions of the

(a)

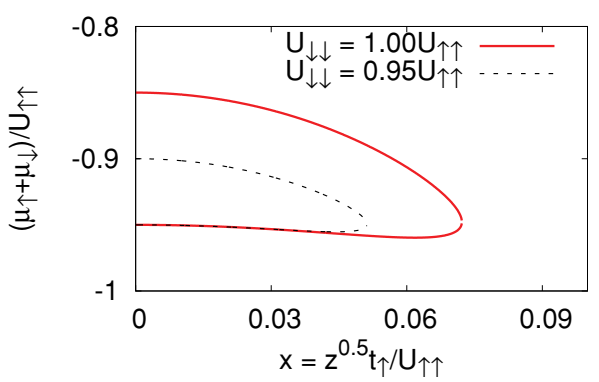

(b)

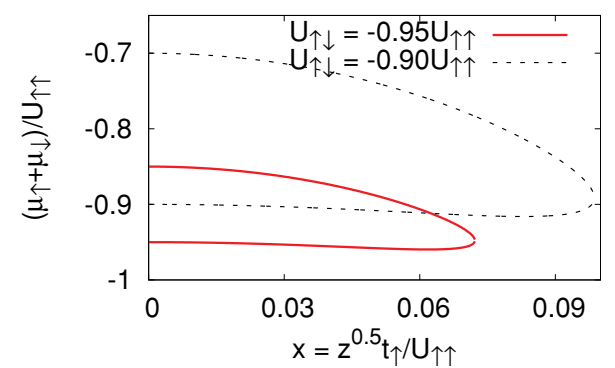

FIG. 1. (Color online) The Mott-insulator-paired-superfluid phase transition boundaries are shown for the first lobe, i.e., $n_{\uparrow}=$ $n_{\downarrow}=1$, when $t_{\uparrow}=t_{\downarrow}$. In (a) $U_{\uparrow \downarrow}=-0.95 U_{\uparrow \uparrow}$, and in (b) $U_{\downarrow \downarrow}=U_{\uparrow \uparrow}$. Note that the red curves correspond to the same data in both figures. two-particle and two-hole excitation energies. Solving Eq. (4) for $\mu_{\uparrow}+\mu_{\downarrow}$, we obtain

$$
\mu_{\uparrow}+\mu_{\downarrow}=\frac{1}{2}\left[a_{\mathrm{p}}-a_{\mathrm{h}} \pm \sqrt{\left(a_{\mathrm{p}}+a_{\mathrm{h}}\right)^{2}-4 c_{\mathrm{p}} c_{\mathrm{h}}}\right],
$$

where $a_{\mathrm{p}}=E_{\mathrm{p}}+\mu_{\uparrow}+\mu_{\downarrow}, a_{\mathrm{h}}=E_{\mathrm{h}}-\mu_{\uparrow}-\mu_{\downarrow}$, and \pm signs correspond to the two-particle and two-hole branches, respectively. Equation (5) is the mean-field expression for the Mott-insulator-paired-superfluid transition boundary, and it is the main result of this paper.

\section{B. Typical phase diagrams}

In this section, we present typical phase diagrams in the $\mu_{\uparrow}+\mu_{\downarrow}$ versus $\sqrt{z} t_{\uparrow}$ plane, obtained directly from Eq. (5). Similar to the usual Bose-Hubbard model, as hopping increases from zero, the range of the chemical potential about which the ground state is a Mott insulator decreases, and the Mott-insulator phase disappears at a critical value of hopping, beyond which the system becomes a paired superfluid.

For instance, in Fig. 1, we show the Mott-insulatorpaired-superfluid phase transition boundaries for the first lobe, i.e., $n_{\uparrow}=n_{\downarrow}=1$, when $t_{\uparrow}=t_{\downarrow}$. In Fig. 1(a), where we set $U_{\uparrow \downarrow}=-0.95 U_{\uparrow \uparrow}$, it is clearly seen that decreasing $U_{\downarrow \downarrow}$ favors the paired-superfluid phase, as intuitively expected. While in Fig. 1(b), where we set $U_{\downarrow \downarrow}=U_{\uparrow \uparrow}$, it is clearly seen that decreasing the strength of $U_{\uparrow \downarrow}$ favors the Mott-insulator phase (see the explanation below). We also note a weak reentrant quantum phase transition in both figures.

In addition, in Fig. 2, we show the Mott-insulator-pairedsuperfluid phase transition boundaries for the first lobe, i.e., $n_{\uparrow}=n_{\downarrow}=1$, when $U_{\downarrow \downarrow}=U_{\uparrow \uparrow}$. In Fig. 2(a), where we set

(a)

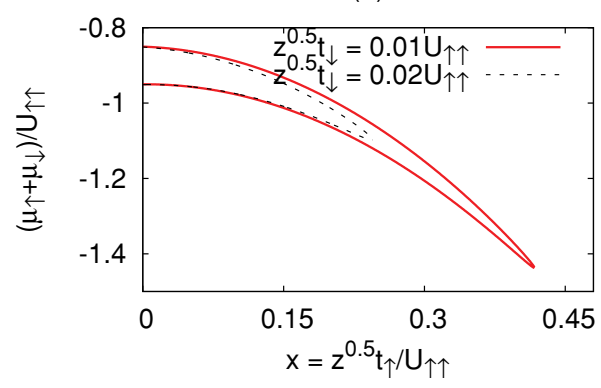

(b)

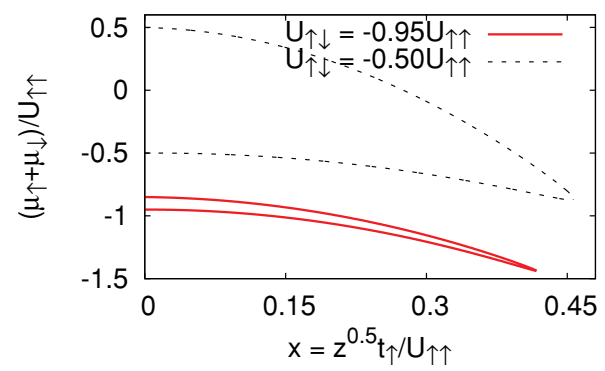

FIG. 2. (Color online) The Mott-insulator-paired-superfluid phase transition boundaries are shown for the first lobe, i.e., $n_{\uparrow}=n_{\downarrow}=1$, when $U_{\downarrow \downarrow}=U_{\uparrow \uparrow}$. In (a) $U_{\uparrow \downarrow}=-0.95 U_{\uparrow \uparrow}$, and in (b) $\sqrt{z} t_{\downarrow}=0.01 U_{\uparrow \uparrow}$. Note that the red curves correspond to the same data in both figures. 
$U_{\uparrow \downarrow}=-0.95 U_{\uparrow \uparrow}$, it is clearly seen that increasing $t_{\downarrow}$ favors the paired-superfluid phase, as intuitively expected. While in Fig. 2(b), where we set $\sqrt{z} t_{\downarrow}=0.01 U_{\uparrow \uparrow}$, it is clearly seen that decreasing the strength of $U_{\uparrow \downarrow}$ again favors the Mott-insulator phase. However, compared to Figs. 1(a) and 1(b), we note that the reentrant quantum phase transition is much stronger in these figures.

Our results are consistent with the expectation that for small $U_{\uparrow \downarrow}$, the location of the Mott-insulator tip increases as a function of $U_{\uparrow \downarrow}$, because the presence of a nonzero $U_{\uparrow \downarrow}$ is what allowed this state to form in the first place. However, when the strength of $U_{\uparrow \downarrow}$ is larger than some critical value (approximately $\sqrt{U_{\uparrow \uparrow} U_{\downarrow \downarrow}} / 2$ ), the location of the tip decreases, and it eventually vanishes exactly when $U_{\uparrow \downarrow}^{2}=$ $U_{\uparrow \uparrow} U_{\downarrow \downarrow}$, which may indicate an instability toward a collapse. In addition, from Eq. (3), we expect a reentrant quantum phase transition when $-\left(2 n_{\uparrow} n_{\downarrow} / U_{\uparrow \downarrow}\right) z t_{\uparrow} t_{\downarrow}-\sum_{\sigma}\left[n_{\sigma}^{2} / U_{\uparrow \downarrow}-\right.$ $\left.\left(n_{\sigma}^{2}-1\right) /\left(2 U_{\sigma \sigma}+U_{\uparrow \downarrow}\right)+2 n_{\sigma}\left(n_{\sigma}+1\right) / U_{\sigma \sigma}\right] z t_{\sigma}^{2}<0$, which occurs beyond a critical $U_{\uparrow \downarrow}$. When this expression is negative, its value is most negative for the first Mott lobe (i.e., $n_{\uparrow}=$ $n_{\downarrow}=1$ ), and therefore the effect is strongest there. However, its value increases and eventually becomes positive as a function of filling, and thus the reentrant behavior becomes weaker as filling increases, and it eventually disappears beyond a critical filling.

\section{CONCLUSIONS}

In this paper, by restricting the two-species Bose-Hubbard Hamiltonian to the subspace of paired particles, and using perturbation theory, we derived an analytic mean-field expression for the Mott-insulator-paired-superfluid phase transition boundary. We found that for small $U_{\uparrow \downarrow}$, the location of the Mott-insulator tip increases as a function of $U_{\uparrow \downarrow}$, because the presence of a nonzero $U_{\uparrow \downarrow}$ is what allowed this state to form in the first place. However, when the strength of $U_{\uparrow \downarrow}$ is larger than some critical value (approximately $\sqrt{U_{\uparrow \uparrow} U_{\downarrow \downarrow}} / 2$ ), the location of the tip decreases, and it eventually vanishes exactly when $U_{\uparrow \downarrow}^{2}=U_{\uparrow \uparrow} U_{\downarrow \downarrow}$, which may indicate an instability toward a collapse. Given that the interspecies interaction can be fine tuned in ongoing experiments, e.g., ${ }^{41} \mathrm{~K}-{ }^{87} \mathrm{Rb}[13,14]$ or homonuclear [15] mixtures, via using Feshbach resonances, we hope that our predictions could be tested with ultracold atomic systems.

\section{ACKNOWLEDGMENTS}

The author thanks Christian Trefzger for correspondence. This work is financially supported by the Marie Curie International Reintegration Grants (FP7-PEOPLE-IRG-2010268239) and Scientific and Technological Research Council of Turkey (TÜBİTAK).
[1] M. Greiner, O. Mandel, T. Esslinger, T. W. Hänsch, and I. Bloch, Nature (London) 415, 39 (2002).

[2] T. Stöferle, H. Moritz, C. Schori, M. Köhl, and T. Esslinger, Phys. Rev. Lett. 92, 130403 (2004).

[3] I. B. Spielman, W. D. Phillips, and J. V. Porto, Phys. Rev. Lett. 98, 080404 (2007).

[4] I. B. Spielman, W. D. Phillips, and J. V. Porto, Phys. Rev. Lett. 100, 120402 (2008).

[5] I. Bloch, J. Dalibard, and W. Zwerger, Rev. Mod. Phys. 80, 885 (2008).

[6] A. B. Kuklov and B. V. Svistunov, Phys. Rev. Lett. 90, 100401 (2003).

[7] E. Altman, W. Hofstetter, E. Demler, and M. D. Lukin, New J. Phys. 5, 113 (2003).
[8] A. Kuklov, N. Prokof'ev, and B. Svistunov, Phys. Rev. Lett. 92, 050402 (2004).

[9] A. Isacsson, M.-C. Cha, K. Sengupta, and S. M. Girvin, Phys. Rev. B 72, 184507 (2005).

[10] A. Argüelles and L. Santos, Phys. Rev. A 75, 053613 (2007).

[11] C. Trefzger, C. Menotti, and M. Lewenstein, Phys. Rev. Lett. 103, 035304 (2009).

[12] M. Iskin, Phys. Rev. A 82, 033630 (2010).

[13] J. Catani, L. De Sarlo, G. Barontini, F. Minardi, and M. Inguscio, Phys. Rev. A 77, 011603(R) (2008).

[14] G. Thalhammer, G. Barontini, L. De Sarlo, J. Catani, F. Minardi, and M. Inguscio, Phys. Rev. Lett. 100, 210402 (2008).

[15] B. Gadway, D. Pertot, R. Reimann, and D. Schneble, Phys. Rev. Lett. 105, 045303 (2010). 